Objective Changes in working conditions give rise to new occupational health risks and work-related diseases (WRDs). Monitoring these new WRDs is essential for their early recognition and prevention and requires a comprehensive approach, using several complementary methods. The aim of this review is to provide an overview and basic typology of different approaches to detect new/emerging WRDs.

Methods We conducted an extensive scientific literature search combining terms for the following three concepts:

- surveillance/reporting systems;

- occupational/work-related diseases; and

- new or emerging risks. In addition, a grey literature search was performed of both grey literature databases and relevant EU and research institute websites for additional resources.

Results We identified a total of 75 surveillance systems from 26 different countries. We set up a basic typology of these systems dividing them into four main groups. Compensationbased systems $(n=22)$ were designed to gather data for compensation purposes and are insurance-driven. Non-compensation-related systems $(n=34)$ were created with the aim of improving the collection and analysis of data to measure trends in occupational and work-related diseases. Sentinel systems $(n=12)$ were specifically designed to provide a warning signal that will initiate health interventions and preventive actions. Finally, public health surveillance systems $(n=7)$ aim to monitor the health of the general population, but can also be used for work-related surveillance. These four main types further differed in terms of disease coverage, means of data collection, evaluation of work-relatedness, follow-up of new/ emerging risks, link with prevention etc.

Conclusion Sentinel systems seem to have the most suitable approach to detect and alert to new/emerging WRDs. Nevertheless, systems identified in the other three groups can also contribute to identifying new/emerging WRDs, despite being primarily designed for other purposes.

\section{6b LESSONS LEARNED FROM EXISTING SENTINEL AND ALERT SYSTEMS}

${ }^{1}$ Annet Lenderink*, ${ }^{2}$ Jelena Bakusic, ${ }^{2}$ Sofie Vandenbroeck, ${ }^{2}$ Charlotte Lambreghts, ${ }^{2}$ Lode Godderis. ${ }^{1}$ Netherlands Centre for Occupational Diseases, Coronel Institute on Work and Health, AMC/University of Amsterdam, Netherlands; '2KULeuven, Occupational, Environmental and Insurance Medicine. Leuven, Belgium

\subsection{6/oemed-2018-ICOHabstracts. 1073}

Introduction Drawing on a literature review on sentinel and alert systems for identifying new/emerging work-related diseases (WRDs) a basic typology of systems was developed. These systems differ in characteristics, ability to capture new WRDs and link with prevention. The objectives of the subsequent study of a subset of systems were to describe in-depth aims, drivers and obstacles of the systems and use of their data in practice, for prevention and detecting new/emerging WRDs.

Methods Twelve systems were chosen reflecting the different types (linked to compensation or not, aimed at all WRDs or a subset of diseases, sentinel systems, workers only or general public). Six systems were described based on desk research and six other systems were studied through interviews with different actors to gather information on the operation of the systems and the use of the gathered data for prevention.

Results Several important themes emerged from the comparative tables, related to the design and performance of the system: visibility, reporting methods, exposure assessment, data quality, linkage to other institutions, and related to data use for prevention, alert on hazardous situations, and awareness on new/emerging diseases.

Conclusion Each system has its strengths and limitations, closely related to its purpose and the country that developed it. Sentinel systems seem to be best equipped for prevention and alert on new/emerging diseases. Enhancing reporting needs to balance required information and perceived reward for reporters. Embedding of systems in governmental or public health organisations is important in terms of financing, expertise and dissemination of results.

\section{C MAREL: THE ITALIAN NETWORK ON WORK-RELATED DISEASES}

${ }^{1} \mathrm{~S}$ Curti* ${ }^{*},{ }^{1} \mathrm{~S}$ Mattioli, ${ }^{2} \mathrm{P}$ Cocco, ${ }^{3} \mathrm{~A}$ Cristaudo, ${ }^{4} \mathrm{M}$ dell'Omo, ${ }^{5} \mathrm{G}$ Mosconi, ${ }^{6} \mathrm{G}$ Campo. ${ }^{1}$ Department of Medical and Surgical Sciences, University of Bologna, Italy; ${ }^{2}$ Department of Public Health, Clinical and Molecular Medicine, University of Cagliari, Italy; ${ }^{3}$ Unit of Occupational Medicine, University of Pisa, Italy; ${ }^{4}$ Unit of Occupational and Environmental Medicine, Department of Medicine, University of Perugia, Italy; ${ }^{5}$ Unit of Occupational Medicine, Azienda Ospedaliera Papa Giovanni XXIII, Bergamo, Italy; ${ }^{6}$ Department of Work and Environmental Medicine, Epidemiology and Hygiene, National Institute for Insurance against Accidents at Work (INAIL), Rome, Italy

\subsection{6/oemed-2018-ICOHabstracts. 1074}

Introduction To study new disease-exposure associations, we created a pilot network (namely MAREL - MAlattie e Rischi Emergenti sul Lavoro) of occupational disease consultation centres of the Italian national health service to which patients are referred for potentially work-related diseases.

Methods The MAREL network included in 2016 five occupational disease consultation centres of university hospitals located in central-northern Italy. Patients were referred to the MAREL consultation centres by their general practitioners, occupational physicians or other specialists for the investigation of the putative occupational origin of a disease. Each centre collected cases of putative occupational origin through a structured and standardised data collection form. We collected data on: diagnosis; personal habits; occupational history; exposure to risk factors; physician's opinion on the possible causal relationship between disease and occupation. Data were coded according to national and international classifications.

Results The data collection started in 2016, enrolling 1516 cases of putative occupational diseases. Musculoskeletal disorders were the most represented conditions: intervertebral lumbar disc degeneration $(11.2 \%)$, spondylitis $(6.3 \%)$, tendinopathies $(4.9 \%)$, arthritis (4.8\%), upper limb mononeuropathies (4.4\%), and shoulder disorders (3.7\%). The most frequently reported exposures were related to biomechanical overload: manual material 